\title{
Subgroups of High-Cost Medicare Advantage Patients: an Observational Study
}

\author{
Brian W. Powers, MD, MBA ${ }^{1,2,3,4}$, Jiali Yan, $M S^{5}$, Jingsan Zhu, MS, MBA ${ }^{6}$, Kristin A. Linn, PhD \\ Sachin H. Jain, MD, MBA ${ }^{3}$, Jennifer L. Kowalski, $M S^{8}$, and Amol S. Navathe, MD, PhD ${ }^{6,9}$
}

'Department of Medicine, Brigham and Women's Hospital, Boston, MA, USA; 'Department of Population Medicine, Harvard Medical School/ Harvard Pilgrim Health Care Institute, Boston, MA, USA; ${ }^{3}$ CareMore Health System, Cerritos, CA, USA; ${ }^{4}$ Atrius Health, Boston, MA, USA; ${ }^{5}$ Department of Medicine, University of Pennsylvania Perelman School of Medicine, Philadelphia, PA, USA; ${ }^{6}$ Department of Medical Ethics and Health Policy, University of Pennsylvania Perelman School of Medicine, Philadelphia, PA, USA; ${ }^{7}$ Department of Biostatistics, Epidemiology and Informatics, University of Pennsylvania Perelman School of Medicine, Philadelphia, PA, USA; ${ }^{8}$ Anthem Public Policy Institute, Washington, DC, USA; ${ }^{9}$ Corporal Michael J. Cresencz VA Medical Center, Philadelphia, PA, USA.

BACKGROUND: There is a growing focus on improving the quality and value of health care delivery for high-cost patients. Compared to fee-for-service Medicare, less is known about the clinical composition of high-cost Medicare Advantage populations.

OBJECTIVE: To describe a high-cost Medicare Advantage population and identify clinically and operationally significant subgroups of patients.

DESIGN:We used a density-based clustering algorithm to group high-cost patients (top 10\% of spending) according to 161 distinct demographic, clinical, and claims-based variables. We then examined rates of utilization, spending, and mortality among subgroups.

PARTICIPANTS: Sixty-one thousand five hundred fortysix Medicare Advantage beneficiaries.

MAIN MEASURES: Spending, utilization, and mortality. KEY RESULTS: High-cost patients $(n=6154)$ accounted for $55 \%$ of total spending. High-cost patients were more likely to be younger, male, and have higher rates of comorbid illnesses. We identified ten subgroups of high-cost patients: acute exacerbations of chronic disease (mixed); end-stage renal disease (ESRD); recurrent gastrointestinal bleed (GIB); orthopedic trauma (trauma); vascular disease (vascular); surgical infections and other complications (complications); cirrhosis with hepatitis C (liver); ESRD with increased medical and behavioral comorbidity (ESRD+); cancer with high-cost imaging and radiation therapy (oncology); and neurologic disorders (neurologic). The average number of inpatient days ranged from 3.25 (oncology) to 26.09 (trauma). Preventable spending (as a percentage of total spending) ranged from $0.8 \%$ (oncology) to $9.5 \%$ (complications) and the percentage of spending attributable to prescription medications ranged from $7.9 \%$ (trauma and oncology) to $77.0 \%$ (liver). The percentage of patients who were persistently high-cost ranged from $11.8 \%$ (trauma) to $100.0 \%$ (ESRD+). One-year mortality ranged from $0.0 \%$ (liver) to $25.8 \%$ (ESRD+).

Electronic supplementary material The online version of this article (https://doi.org/10.1007/s11606-018-4759-1) contains supplementary material, which is available to authorized users.

Received July 24, 2018

Revised September 14, 2018

Accepted November 16, 2018

Published online December 3, 2018
CONCLUSIONS: We identified clinically distinct subgroups of patients within a heterogeneous high-cost Medicare Advantage population using cluster analysis. These subgroups, defined by condition-specific profiles and illness trajectories, had markedly different patterns of utilization, spending, and mortality, holding important implications for clinical strategy.

KEY WORDS: high-cost patients; care management; medicare advantage.

$\mathrm{J}$ Gen Intern Med 34(2):218-25

DOI: $10.1007 / \mathrm{s} 11606-018-4759-1$

(C) Society of General Internal Medicine 2018

\section{INTRODUCTION}

The concentration of health care spending - $5 \%$ of patients incur roughly half of total spending ${ }^{1-3}$ - has prompted an intense focus on improving the quality and value of health care delivery for high-cost patients. ${ }^{3-5}$ Current efforts to describe and segment high-cost patients have focused predominately on fee-for-service (FFS) Medicare populations. ${ }^{3,6,7}$ Despite the fact that one in three Medicare beneficiaries is now enrolled in a Medicare Advantage (MA) plan, ${ }^{8}$ less is known about high-cost MA patients.

Better understanding the clinical composition of high-cost patient populations has the potential to improve care management program design. Researchers and policymakers have suggested that suboptimal patient targeting-singular interventions are frequently applied to diverse patient populations - may be driving the limited effectiveness of current care management approaches. $3,7,9,10$

Descriptive analyses have shown that within high-cost patient populations, there is substantial variation in demographics, functional status, diagnoses, and disease burden. , $^{6,11-13}$ Improving care management program effectiveness, therefore, may require identifying distinct subgroups of high-cost patients, and tailoring interventions to meet the unique needs of each group. Existing frameworks for identifying subgroups of high-cost patients are derived from expert opinion. 3,6,7,14 There may be an opportunity to supplement these approaches 
by identifying subgroups exclusively based on the analysis of variation within patient data.

In this study, we aimed to (1) describe the demographic and clinical characteristics of a high-cost MA population, (2) use cluster analysis to derive high-cost patient subgroups from large volumes of clinical and claims data, and (3) explore whether these subgroups were meaningfully associated with patterns of utilization, spending, and mortality.

\section{METHODS}

\section{Study Population}

We obtained data for patients enrolled in MA plans offered by CareMore Health System in $2014(n=93,047)$. CareMore, a subsidiary of Anthem, Inc., offered MA plans in California, Arizona, and Virginia in 2014. We excluded patients who were not continuously enrolled for the entire year $(n=27,163)$, those who died during $2014(n=3649)$, those who did not have any approved medical claims during $2014(n=1867)$, and those enrolled in an institutional special needs plan $(n=$ 1981). The final study population consisted of 61,546 patients.

\section{Data}

We extracted demographic, clinical, utilization, spending, and mortality data for the study population from CareMore's electronic data warehouse (EDW). The EDW aggregates data from electronic medical records (EMR) and administrative sources. Data were obtained for the years 2013-2015.

A full description of study variables is provided in Appendix 1. Briefly, we grouped variables into the following categories: Demographics, chronic conditions, active diagnoses, procedures, laboratory, pharmacy, mortality, utilization, and spending. Demographic variables included age and gender. Chronic conditions were assessed individually according to the Elixhauser ${ }^{15}$ classification using prior year (2013) ICD-9 diagnosis codes. Active diagnoses were determined using 2014 ICD-9 codes, and grouped according to Agency for Healthcare Research and Quality (AHRQ) Clinical Conditions Software (CCS) categories. ${ }^{16}$ Procedures were determined using 2014 CPT codes, and grouped according to AHRQ CSS categories. ${ }^{17}$ Laboratory variables were assessed directly from the EMR. Pharmacy variables were calculated using pharmacy fill data, and included the number of unique medications as well as adherence, which was defined as the proportion of days covered $(\mathrm{PDC})^{18}$ among a limited set of common outpatient medications. Subsequent year (2015) mortality was assessed directly from the EDW. Utilization and spending variables were assessed directly from paid claims. Preventable spending was calculated using the AHRQ Prevention Quality Indicators algorithm ${ }^{19}$ for inpatient spending, and the algorithm created by Billings et al. ${ }^{20}$ for emergency department spending, both of which have been validated and used in prior work segmenting high-cost Medicare populations. ${ }^{21,22}$

\section{Descriptive Analyses}

We defined "high-cost" patients as those in the top decile of spending in 2014 ( $n=6154)$. First, we described demographic, chronic condition, pharmacy, utilization, and spending variables across the entire study population. We then compared these variables between high-cost and non-high-cost subgroups using $t$ tests for continuous variables and $\chi^{2}$ tests for categorical variables.

\section{Identifying Subgroups}

Clustering is an unsupervised machine learning technique that groups observations (e.g., patients) according to similarities among measured characteristics. Clustering algorithms iteratively group observations into subgroups until finding the allocation that maximizes both intra-group similarity and inter-group differences (see an accompanying article ${ }^{23}$ for more information).

The dataset used for cluster analysis included demographics, chronic conditions, active diagnoses, procedures, laboratory, and pharmacy variables for the subset of high-cost patients $(n=6154)$. Utilization and spending variables were not used for clustering, allowing for comparison of utilization and spending across clusters (see below).

To perform cluster analysis, we began by analytically reducing the number of variables in the dataset - a task known in computer science as dimension reduction. We removed variables with extremely low variance, and those that were highly correlated. A total of 161 variables remained (full list provided in Appendix 2). We then utilized a non-linear dimension reduction algorithm ${ }^{24,25}$ to create a low-dimension representation of the dataset. Additional detail regarding dimension reduction is provided in Appendix 3 and an accompanying article. ${ }^{23}$ Finally, we applied a density-based clustering algorithm-Ordering Points To Identify the Clustering Structure (OPTICS) ${ }^{26,27}$ - to the low-dimension dataset. We restricted the minimum number of patients per subgroup to be at least 62 (or $1 \%$ of the high-cost population) in order to ensure that the subgroups were operationally meaningful. Our rationale for choosing the OPTICS algorithm and information on tuning parameters is described in an accompanying article. ${ }^{23}$

\section{Subgroup Analysis}

To describe the clinical composition of the resultant subgroups, we first calculated high-cost population means and subgroupspecific means for each variable used in clustering. We then calculated standardized ratios of subgroup means to population means, such that larger numbers represented variables for which the subgroup deviated most from the broader highcost population. We assigned a clinical descriptive label to each subgroup based on the variables with the highest standardized ratios as well as variables for which the ratios varied most among subgroups. Given the numerous variables, we chose to present the ten variables with the largest standardized ratios (labeled as "distinguishing factors") for each subgroup. Appendix 4 contains a complete list of standardized ratios for all subgroups. 
Next, we compared spending, utilization, and mortality across subgroups. We calculated 2014 utilization rates, average spending, composition of spending, and rates of preventable spending among each subgroup. To better understand the trajectory of spending for subgroups, we calculated average spending, preventable spending, and the prevalence of persistent high-cost status in 2015. We defined persistent high-cost status as remaining in the top decile of total spending in 2015. Finally, we calculated 2015 mortality rates among each subgroup. We excluded patients not continuously enrolled in a CareMore MA plan from the 2015 analyses $(n=1430)$.

Data preparations were done in SAS version 9.4 (SAS Institute, Cary, NC). R version 3.2.5 was used for all other analyses.

\section{RESULTS}

\section{High-Cost Patient Characteristics}

In a national Medicare Advantage population $(n=61,546)$, the highest cost $10 \%$ of patients $(n=6154)$ accounted for $55 \%$ of total population spending in 2014, with average annual spending of $\$ 55,696$ per patient. Among high-cost patients in 2014, $64 \%$ were persistently high-cost (remained in top $10 \%$ of spending in 2015).

Table 1 describes utilization patterns for high-cost and nonhigh-cost patients. Compared to non-high-cost patients, highcost patients had higher average rates of inpatient (IP) admissions (1.7 vs. 0.1$)$, IP days (12.9 vs. 0.9), and emergency department (ED) visits (2.4 vs. 0.4). The total annual spending was roughly ten times higher among high-cost patients (\$55,696 vs. $\$ 5071$ ), and the rate of preventable spending was also notably higher (7.1\% vs. $3.6 \%)$.

High-cost patients also varied substantially from non-highcost patients across demographic and clinical characteristics (Table 1). High-cost patients were younger (average age 70.9 vs. 73.5 ), and more likely to be male ( $49.6 \%$ vs. $42.9 \%$ ). Highcost patient had higher rates of co-occurring chronic conditions (9.0 vs. 4.4) as well as significantly higher rates of all individually assessed chronic conditions, including congestive heart failure ( $44 \%$ vs. $11 \%$ ), vascular disease (60\% vs. $28 \%)$, and renal failure (69\% vs. 39\%). Among high-cost patients, rates of polypharmacy were higher (14.6 vs. 7.9 average prescriptions), and medication adherence was lower (0.7 vs. 0.8 PDC).

\section{High-Cost Patient Subgroups}

Cluster analysis identified ten subgroups of high-cost patients. The number of patients in each subgroup ranged from 56 to 3686, and 382 patients were not assigned to any subgroup. Differentiation across subgroups was driven predominately by comorbidities and procedures; laboratory values and demographics were less important (Table 2). There were divergent patterns of index year utilization and spending (Table 3) as well as spending and mortality trajectories (Table 4) across subgroups. Each subgroup and associated patterns of utilization, spending, and mortality are described below. Comparative statements are relative to other high-cost patients, not the entire study population.

Acute Exacerbations of Chronic Disease (Mixed). Distinguished by procedures associated with acute hospitalizations. These patients also had higher-than-average rates of cerebrovascular disease, chronic obstructive pulmonary disease, ischemic heart disease, congestive heart failure, and behavioral health disorders. Although rates of utilization and spending were close to average, this subgroup was characterized by higher-thanaverage rates of preventable spending in 2014 (7.9\%) and $2015(4.3 \%)$.

End-Stage Renal Disease. Distinguished by active diagnoses, chronic conditions, and procedures related to end-stage renal disease (ESRD) and dialysis. These patients were among the highest cost (average 2014 spending \$74,385), were among the most likely to be persistently high-cost (97.3\%), and had a high rate of mortality in 2015 (18.5\%). Inpatient and ED utilization were substantially below average.

Recurrent Gastrointestinal Bleed. Distinguished by active diagnoses and procedures related to recurrent gastrointestinal bleed (GIB), as well as chronic conditions representing the sequelae of recurrent GIB. Rates of ED and inpatient utilization were among the highest (2.74 inpatient admissions, $3.25 \mathrm{ED}$ admissions, and 18.96 inpatient days) in 2014, but these patients were among the least likely to remain persistently high-cost (34.6\%).

Orthopedic Trauma (Trauma). Distinguished by active diagnoses and procedures related to fractures and other traumatic events. These patients had the highest rates of inpatient utilization (26.09 inpatient days), but were the least likely to be persistently high-cost (11.8\%).

Vascular Disease (Vascular). Distinguished by active diagnoses and procedures related to peripheral vascular disease. Rates of utilization and spending in 2014 were among the lowest; rates of persistently high-cost status were close to average.

Surgical Infections and Other Complications (Complications). Distinguished by active diagnoses and procedures related to surgical wounds, infections, and other iatrogenic complications. Patients in this subgroup had higherthan-average rates of inpatient utilization and total spending (19.69 inpatient days and total spending of $\$ 60,103)$, but were less likely to be persistently high-cost $(31.1 \%)$. The rate of mortality in 2015 was among the highest (19.7\%).

Cirrhosis with Hepatitis $C$ (Liver). Distinguished by active diagnoses and chronic conditions related to the diagnosis, 
TABLE 1 Characteristics of the Study Population, by High-Cost Status

\begin{tabular}{|c|c|c|c|c|}
\hline & Overall population & High-cost subgroup & Non-high-cost subgroup & $p$ value \\
\hline Number of patients (no.) & 61,546 & 6155 & 55,391 & \\
\hline \multicolumn{5}{|l|}{ Demographics } \\
\hline Age, mean (SD) & $73.2(9.5)$ & $70.9(11.4)$ & $73.5(9.2)$ & $<0.001$ \\
\hline Male $(\%)$ & 43.5 & 49.6 & 42.9 & $<0.001$ \\
\hline \multicolumn{5}{|l|}{ Chronic conditions } \\
\hline Total no., mean (SD) & $4.9(3.2)$ & $9(3.4)$ & $4.4(2.8)$ & $<0.001$ \\
\hline Hypertension (\%) & 80.4 & 94.4 & 78.9 & $<0.001$ \\
\hline Renal failure $(\%)$ & 42.3 & 69.0 & 39.4 & $<0.001$ \\
\hline Diabetes uncomplicated $(\%)$ & 41.5 & 63.1 & 39.1 & $<0.001$ \\
\hline Diabetes complicated (\%) & 38.4 & 59.3 & 36.0 & $<0.001$ \\
\hline Obesity $(\%)$ & 31.9 & 44.6 & 30.5 & $<0.001$ \\
\hline Peripheral vascular disorders $(\%)$ & 31.2 & 60.4 & 28.0 & $<0.001$ \\
\hline Depression $(\%)$ & 30.2 & 46.1 & 28.4 & $<0.001$ \\
\hline Chronic pulmonary disease $(\%)$ & 28.8 & 48.4 & 26.6 & $<0.001$ \\
\hline Hypothyroidism $(\%)$ & 21.3 & 27.7 & 20.6 & $<0.001$ \\
\hline Drug abuse $(\%)$ & 19.7 & 37.0 & 17.7 & $<0.001$ \\
\hline Fluid and electrolyte disorders (\%) & 16.9 & 54.8 & 12.7 & $<0.001$ \\
\hline Congestive heart failure $(\%)$ & 14.3 & 43.9 & 11.0 & $<0.001$ \\
\hline Weight loss $(\%)$ & 12.4 & 37.5 & 9.6 & $<0.001$ \\
\hline Valvular disease $(\%)$ & 11.5 & 28.2 & 9.6 & $<0.001$ \\
\hline Solid tumor without metastasis $(\%)$ & 9.1 & 19.0 & 8.0 & $<0.001$ \\
\hline Rheumatoid arthritis/collagen (\%) & 9.0 & 15.4 & 8.3 & $<0.001$ \\
\hline Other neurological disorders $(\%)$ & 8.6 & 23.0 & 7.1 & $<0.001$ \\
\hline Deficiency anemia $(\%)$ & 8.0 & 33.1 & 5.2 & $<0.001$ \\
\hline Liver disease $(\%)$ & 7.6 & 17.1 & 6.5 & $<0.001$ \\
\hline Coagulopathy (\%) & 5.5 & 19.7 & 3.9 & $<0.001$ \\
\hline Alcohol abuse (\%) & 5.3 & 9.7 & 4.8 & $<0.001$ \\
\hline Psychoses $(\%)$ & 3.4 & 9.2 & 2.8 & $<0.001$ \\
\hline Pulmonary circulation disorders $(\%)$ & 3.4 & 11.3 & 2.5 & $<0.001$ \\
\hline Peptic ulcer disease $(\%)$ & 2.1 & 5.6 & 1.8 & $<0.001$ \\
\hline Blood loss anemia (\%) & 1.6 & 6.7 & 1.1 & $<0.001$ \\
\hline Paralysis $(\%)$ & 1.6 & 5.3 & 1.2 & $<0.001$ \\
\hline Metastatic cancer $(\%)$ & 1.4 & 6.4 & 0.9 & $<0.001$ \\
\hline Lymphoma $(\%)$ & 0.8 & 2.6 & 0.6 & $<0.001$ \\
\hline AIDS/HIV (\%) & 0.1 & 0.7 & 0.0 & $<0.001$ \\
\hline \multicolumn{5}{|l|}{ Prescription medications } \\
\hline No. of prescriptions, mean (SD) & $8.5(5.5)$ & $14.6(6.3)$ & $7.9(4.9)$ & $<0.001$ \\
\hline Proportion of days covered, mean (SD) & $0.8(0.2)$ & $0.7(0.2)$ & $0.8(0.2)$ & $<0.001$ \\
\hline \multicolumn{5}{|l|}{ Utilization } \\
\hline Inpatient admissions, mean (SD) & $0.3(0.8)$ & $1.7(1.8)$ & $0.1(0.4)$ & $<0.001$ \\
\hline Inpatient days, mean (SD) & $2.1(100.4)$ & $12.9(20.8)$ & $0.9(105.6)$ & $<0.001$ \\
\hline Emergency department visits, mean (SD) & $0.6(1.6)$ & $2.4(3.7)$ & $0.4(0.9)$ & $<0.001$ \\
\hline Total spending $(\$)$, mean $(\mathrm{SD})$ & $10,133(19,842)$ & $55,696(37,217)$ & $5071(5229)$ & $<0.001$ \\
\hline Preventable spending $(\$)$, mean (SD) & $558(4114)$ & $3930(12,168)$ & $184(980)$ & $<0.001$ \\
\hline
\end{tabular}

SD, standard deviation; AIDS/HIV, acquired immunodeficiency syndrome/human immunodeficiency virus

management, and sequelae of hepatitis $\mathrm{C}$ infection. These patients had the highest rates of average spending in 2014 $(\$ 78,706)$, which was driven predominately by prescription drug costs (77.0\% of total spending). Patients in this subgroup were more likely than average to remain high-cost $(58.6 \%)$ and had the lowest rate of mortality in $2015(0.0 \%)$.

\section{ESRD with Increased Medical and Behavioral Comorbidity} (ESRD+). Distinguished by diagnoses, chronic conditions, and procedures related to ESRD and dialysis. Compared to the ESRD subgroup, there were higher rates of congestive heart failure, behavioral health disorders, liver failure, and cerebrovascular disease. Patients in this subgroup also had higher rates of preventable spending ( $4.8 \%$ vs. $3.0 \%)$ than those in the ESRD subgroup. These patients were the most likely to remain high-cost in $2015(100.0 \%)$ and had the highest rate of mortality in 2015 (25.8\%).

Cancer with High-Cost Imaging and Radiation Therapy (Oncology). Distinguished by metastatic and non-metastatic cancer diagnoses, imaging procedures related to disease staging and surveillance, and brachytherapy. Patients were predominately male $(88 \%)$ and the most common oncologic diagnosis was prostate cancer. Rates of 2014 utilization (3.25 inpatient days), spending $(\$ 46,240)$, persistent high-cost status $(20.0 \%)$, and mortality in $2015(5.5 \%)$ were among the lowest.

Neurologic Disorders (Neurologic). Distinguished by active diagnoses and chronic conditions encompassing neurologic disorders (most notably multiple sclerosis) and neurologic diagnostic procedures. These patients had among the lowest rates of 2014 inpatient utilization, but were among the most likely to remain high-cost $(75.6 \%)$, with spending driven by prescription drugs ( $51.7 \%$ of total spending).

\section{DISCUSSION}

We found that health care spending was highly concentrated in a national Medicare Advantage population. The highest cost 
TABLE 2 Description of High-Cost Patient Subgroups

\begin{tabular}{|c|c|c|c|}
\hline \multirow[t]{2}{*}{ Description } & \multirow[t]{2}{*}{ No. of patients (\%) } & \multicolumn{2}{|l|}{ Distinguishing factors } \\
\hline & & Variable* & Category \\
\hline $\begin{array}{l}\text { Acute exacerbations of chronic } \\
\text { disease } \\
\text { (mixed) }\end{array}$ & $\begin{array}{l}3686 \\
(59.9 \%)\end{array}$ & $\begin{array}{l}\text { Arterial blood gases } \\
\text { Bacterial infection } \\
\text { Metastatic cancer } \\
\text { Other vascular catheterization; not heart } \\
\text { CT scan chest } \\
\text { Routine chest X-ray } \\
\text { Electroencephalogram } \\
\text { Other respiratory therapy } \\
\text { Cerebrovascular disease } \\
\text { CT scan abdomen }\end{array}$ & $\begin{array}{l}\text { Procedures } \\
\text { Active diagnoses } \\
\text { Chronic conditions } \\
\text { Procedures } \\
\text { Procedures } \\
\text { Procedures } \\
\text { Procedures } \\
\text { Procedures } \\
\text { Active diagnoses } \\
\text { Procedures }\end{array}$ \\
\hline $\begin{array}{l}\text { End-stage renal } \\
\text { disease } \\
\text { (ESRD) }\end{array}$ & $\begin{array}{l}1222 \\
(19.9 \%)\end{array}$ & $\begin{array}{l}\text { Peritoneal dialysis } \\
\text { Diseases of the urinary system } \\
\text { Complications } \\
\text { Iron deficiency anemia } \\
\text { Other vascular catheterization; not heart } \\
\text { Creatinine } \\
\text { Bacterial infection } \\
\text { Fluid and electrolyte disorders } \\
\text { Blood loss anemia } \\
\text { Congestive heart failure }\end{array}$ & $\begin{array}{l}\text { Procedures } \\
\text { Active diagnoses } \\
\text { Active diagnoses } \\
\text { Chronic conditions } \\
\text { Procedures } \\
\text { Laboratory } \\
\text { Active diagnoses } \\
\text { Chronic conditions } \\
\text { Chronic conditions } \\
\text { Chronic conditions }\end{array}$ \\
\hline $\begin{array}{l}\text { Recurrent GI } \\
\text { bleed } \\
\text { (GIB) }\end{array}$ & $\begin{array}{l}221 \\
(3.6 \%)\end{array}$ & $\begin{array}{l}\text { Blood loss anemia } \\
\text { Gastrointestinal hemorrhage } \\
\text { Peptic ulcer disease excluding bleeding } \\
\text { Upper gastrointestinal endoscopy; biopsy } \\
\text { Anemia } \\
\text { Other vascular catheterization; not heart } \\
\text { Iron deficiency anemia } \\
\text { Coagulopathy } \\
\text { Routine chest X-ray } \\
\text { CT scan abdomen }\end{array}$ & $\begin{array}{l}\text { Chronic conditions } \\
\text { Active diagnoses } \\
\text { Chronic conditions } \\
\text { Procedures } \\
\text { Active diagnoses } \\
\text { Procedures } \\
\text { Chronic conditions } \\
\text { Chronic conditions } \\
\text { Procedures } \\
\text { Procedures }\end{array}$ \\
\hline $\begin{array}{l}\text { Orthopedic trauma } \\
\text { (trauma) }\end{array}$ & $\begin{array}{l}201 \\
(3.3 \%)\end{array}$ & $\begin{array}{l}\text { Fractures } \\
\text { Headache } \\
\text { Traction; splints; and other wound care } \\
\text { Other diagnostic radiology } \\
\text { Other CT scan } \\
\text { Other injuries/conditions due to external causes } \\
\text { Other non-OR nervous system procedures } \\
\text { Diagnostic physical therapy } \\
\text { Electroencephalogram } \\
\text { CT scan head }\end{array}$ & $\begin{array}{l}\text { Active diagnoses } \\
\text { Active diagnoses } \\
\text { Procedures } \\
\text { Procedures } \\
\text { Procedures } \\
\text { Active diagnoses } \\
\text { Procedures } \\
\text { Procedures } \\
\text { Procedures } \\
\text { Procedures }\end{array}$ \\
\hline $\begin{array}{l}\text { Vascular disease with } \\
\text { interventions (vascular) }\end{array}$ & $\begin{array}{l}86 \\
(1.4 \%)\end{array}$ & $\begin{array}{l}\text { Diseases of arteries; arterioles; and capillaries } \\
\text { Other vascular catheterization; not heart } \\
\text { Other diagnostic cardiovascular procedures } \\
\text { Complications } \\
\text { Diseases of veins and lymphatics } \\
\text { History of mental health/substance abuse } \\
\text { Diagnostic US } \\
\text { Open wounds } \\
\text { Anesthesia } \\
\text { Cerebrovascular disease }\end{array}$ & $\begin{array}{l}\text { Active diagnoses } \\
\text { Procedures } \\
\text { Procedures } \\
\text { Active diagnoses } \\
\text { Active diagnoses } \\
\text { Active diagnoses } \\
\text { Procedures } \\
\text { Active diagnoses } \\
\text { Procedures } \\
\text { Active diagnoses }\end{array}$ \\
\hline $\begin{array}{l}\text { Surgical infections and } \\
\text { other complications } \\
\text { (complications) }\end{array}$ & $\begin{array}{l}81 \\
(1.3 \%)\end{array}$ & $\begin{array}{l}\text { Complications } \\
\text { Traction; splints; and other wound care } \\
\text { Open wounds } \\
\text { Ancillary services } \\
\text { Other vascular catheterization; not heart } \\
\text { Skin and subcutaneous tissue infections } \\
\text { Blood loss anemia } \\
\text { Anesthesia } \\
\text { Bacterial infection } \\
\text { Electroencephalogram }\end{array}$ & $\begin{array}{l}\text { Active diagnoses } \\
\text { Procedures } \\
\text { Active diagnoses } \\
\text { Procedures } \\
\text { Procedures } \\
\text { Active diagnoses } \\
\text { Chronic conditions } \\
\text { Procedures } \\
\text { Active diagnoses } \\
\text { Procedures }\end{array}$ \\
\hline $\begin{array}{l}\text { Cirrhosis with hepatitis C } \\
\text { (liver) }\end{array}$ & $\begin{array}{l}81 \\
(1.3 \%)\end{array}$ & $\begin{array}{l}\text { Viral infection } \\
\text { Liver disease } \\
\text { Liver disease } \\
\text { Upper gastrointestinal endoscopy; biopsy } \\
\text { Other bone diseases and musculoskeletal deformities } \\
\text { Radioisotope scan and function studies } \\
\text { Immunizations and screening for infectious disease } \\
\text { Anxiety disorders } \\
\text { Coagulopathy } \\
\text { Other injuries and conditions due to external causes }\end{array}$ & $\begin{array}{l}\text { Active diagnoses } \\
\text { Active diagnoses } \\
\text { Chronic conditions } \\
\text { Procedures } \\
\text { Active diagnoses } \\
\text { Procedures } \\
\text { Active diagnoses } \\
\text { Active diagnoses } \\
\text { Chronic conditions } \\
\text { Active diagnoses }\end{array}$ \\
\hline
\end{tabular}


TABLE 2. (continued)

\begin{tabular}{|c|c|c|c|}
\hline \multirow[t]{2}{*}{ Description } & \multirow[t]{2}{*}{ No. of patients (\%) } & \multicolumn{2}{|l|}{ Distinguishing factors } \\
\hline & & Variable* & Category \\
\hline $\begin{array}{l}\text { ESRD with increased medical } \\
\text { and behavioral comorbidity } \\
(\text { ESRD+) }\end{array}$ & $\begin{array}{l}73 \\
(1.2 \%)\end{array}$ & $\begin{array}{l}\text { Peritoneal dialysis } \\
\text { Diseases of the urinary system } \\
\text { Complications } \\
\text { Iron deficiency anemia } \\
\text { Other vascular catheterization; not heart } \\
\text { Blood loss anemia } \\
\text { Creatinine } \\
\text { Congestive heart failure } \\
\text { Coagulopathy } \\
\text { Fluid and electrolyte disorders }\end{array}$ & $\begin{array}{l}\text { Procedures } \\
\text { Active diagnoses } \\
\text { Active diagnoses } \\
\text { Chronic conditions } \\
\text { Procedures } \\
\text { Chronic conditions } \\
\text { Laboratory } \\
\text { Chronic conditions } \\
\text { Chronic conditions } \\
\text { Chronic conditions }\end{array}$ \\
\hline $\begin{array}{l}\text { Cancer with high-cost } \\
\text { Imaging and radiation } \\
\text { therapy (oncology) }\end{array}$ & $\begin{array}{l}65 \\
(1.1 \%)\end{array}$ & $\begin{array}{l}\text { Other CT scan } \\
\text { Metastatic cancer } \\
\text { Solid tumor without metastasis } \\
\text { Diagnostic procedures on nose; mouth; and pharynx } \\
\text { Radioisotope scan and function studies } \\
\text { Fluid and electrolyte disorders } \\
\text { Diseases of male genital organs } \\
\text { Magnetic resonance imaging } \\
\text { CT scan abdomen } \\
\text { Noninfectious gastroenteritis }\end{array}$ & $\begin{array}{l}\text { Procedures } \\
\text { Chronic conditions } \\
\text { Chronic conditions } \\
\text { Procedures } \\
\text { Procedures } \\
\text { Active diagnoses } \\
\text { Active diagnoses } \\
\text { Procedures } \\
\text { Procedures } \\
\text { Active diagnoses }\end{array}$ \\
\hline $\begin{array}{l}\text { Neurologic } \\
\text { disorders(neurologic) }\end{array}$ & $\begin{array}{l}56 \\
(0.9 \%)\end{array}$ & $\begin{array}{l}\text { Hereditary/degenerative nervous system conditions } \\
\text { Other neurological disorders } \\
\text { Electroencephalogram } \\
\text { CT scan head } \\
\text { Paralysis } \\
\text { Magnetic resonance imaging } \\
\text { Noninfectious gastroenteritis } \\
\text { Bacterial infection } \\
\text { Diagnostic physical therapy } \\
\text { Psychoses }\end{array}$ & $\begin{array}{l}\text { Active diagnoses } \\
\text { Chronic conditions } \\
\text { Procedures } \\
\text { Procedures } \\
\text { Chronic conditions } \\
\text { Procedures } \\
\text { Active diagnoses } \\
\text { Active diagnoses } \\
\text { Procedures } \\
\text { Chronic conditions }\end{array}$ \\
\hline
\end{tabular}

ESRD, end-stage renal disease, GIB, gastrointestinal bleed; CT, computed tomography; OR, operating room

*As described in the "Methods" section, these variables represent those with the highest standardized ratios (subgroup means to high-cost population means), represented in descending order (see Appendix 4 for a complete list of all standardized ratios for all variables among all subgroups)

$10 \%$ of patients accounted for $55 \%$ of total spending, a level of spending concentration similar to that of FFS Medicare., 28 The majority of high-cost patients were persistently highcost $-65 \%$ remained in the highest cost decile the following year. This is in contrast to FFS Medicare, where rates of persistently high-cost status range from 25 to $45 \%$. $^{2,21,28}$

High-cost patients in this study had roughly twice as many co-occurring chronic conditions as non-high-cost patients, which is consistent with recent research in a FFS Medicare population. ${ }^{7}$ High-cost patients had higher rates of all comorbid conditions assessed, including diabetes, congestive heart failure, chronic obstructive pulmonary disease, hypertension, depression, and renal failure. Rates of comorbid conditions were higher than those previously described for high-cost FFS Medicare beneficiaries. ${ }^{2,21,28,29}$

To better understand the composition of this high-cost population, we used cluster analysis to identify subgroups of patients according to similarities across 161 demographic and clinical variables. We identified ten subgroups: acute exacerbations of chronic disease (mixed); end-stage renal disease (ESRD); recurrent gastrointestinal bleed (GIB); orthopedic trauma (trauma); vascular disease (vascular); surgical infections and other complications (complications); cirrhosis with hepatitis C (liver); ESRD with increased medical and behavioral comorbidity (ESRD+); cancer with high-cost imaging and radiation therapy (oncology); and neurologic

TABLE 3 Utilization and Spending for High-Cost Patient Subgroups, 2014

\begin{tabular}{|c|c|c|c|c|c|c|c|c|c|c|}
\hline & Mixed & ESRD & GIB & Trauma & Vascular & Complications & Liver & ESRD+ & Oncology & Neurologic \\
\hline $\begin{array}{l}\text { Inpatient admissions } \\
\text { (mean) }\end{array}$ & 1.87 & 1.01 & 2.74 & 2.42 & 1.49 & 2.46 & 0.52 & 1.58 & 0.4 & 1.39 \\
\hline Inpatient days (mean) & 14.4 & 6.07 & 18.96 & 26.09 & 9.33 & 19.69 & 3.98 & 8.47 & 3.25 & 14.5 \\
\hline ED visits (mean) & 2.59 & 1.94 & 3.25 & 2.65 & 1.28 & 2.1 & 0.91 & 2.3 & 0.75 & 1.77 \\
\hline Total spending, mean $(\$)$ & 47,936 & 74,385 & 52,259 & 40,175 & 45,829 & 60,103 & 78,706 & 79,724 & 46,240 & 66,417 \\
\hline Preventable (\%) & $7.9 \%$ & $3.0 \%$ & $8.0 \%$ & $5.7 \%$ & $6.8 \%$ & $9.5 \%$ & $0.9 \%$ & $4.8 \%$ & $0.8 \%$ & $3.8 \%$ \\
\hline Inpatient $(\%)$ & $42.0 \%$ & $12.8 \%$ & $57.2 \%$ & $44.2 \%$ & $42.7 \%$ & $40.2 \%$ & $9.4 \%$ & $13.0 \%$ & $4.4 \%$ & $20.4 \%$ \\
\hline Medication (\%) & $18.4 \%$ & $11.1 \%$ & $10.9 \%$ & $7.9 \%$ & $14.2 \%$ & $11.3 \%$ & $77.0 \%$ & $8.1 \%$ & $7.9 \%$ & $51.7 \%$ \\
\hline Other $(\%)$ & $39.6 \%$ & $76.1 \%$ & $31.9 \%$ & $47.9 \%$ & $43.1 \%$ & $48.5 \%$ & $13.6 \%$ & $78.8 \%$ & $87.7 \%$ & $27.9 \%$ \\
\hline
\end{tabular}

ESRD, end-stage renal disease; GIB, gastrointestinal bleed; ED, emergency department 
TABLE 4 Utilization and Spending Trajectories for High-Cost Patient Subgroups, 2014-2015

\begin{tabular}{|c|c|c|c|c|c|c|c|c|}
\hline & \multicolumn{2}{|c|}{ Total spending, mean (\$) } & \multicolumn{2}{|c|}{$\begin{array}{l}\text { Preventable } \\
\text { spending }(\%)\end{array}$} & \multicolumn{2}{|c|}{$\begin{array}{l}\text { Top decile of total } \\
\text { spending }(\%)\end{array}$} & \multicolumn{2}{|c|}{ Mortality* (\%) } \\
\hline & 2014 & 2015 & 2014 & 2015 & 2014 & 2015 & 2014 & 2015 \\
\hline Mixed & $\$ 47,936$ & $\$ 27,513$ & $7.9 \%$ & $4.3 \%$ & $100.0 \%$ & $35.8 \%$ & - & $13.5 \%$ \\
\hline ESRD & $\$ 74,385$ & $\$ 80,245$ & $3.0 \%$ & $2.1 \%$ & $100.0 \%$ & $97.3 \%$ & - & $18.5 \%$ \\
\hline GIB & $\$ 52,259$ & $\$ 25,985$ & $8.0 \%$ & $4.1 \%$ & $100.0 \%$ & $34.6 \%$ & - & $17.0 \%$ \\
\hline Trauma & $\$ 40,175$ & $\$ 13,685$ & $5.7 \%$ & $4.1 \%$ & $100.0 \%$ & $11.8 \%$ & - & $10.4 \%$ \\
\hline Vascular & $\$ 45,829$ & $\$ 29,044$ & $6.8 \%$ & $2.1 \%$ & $100.0 \%$ & $35.7 \%$ & - & $8.6 \%$ \\
\hline Complications & $\$ 60,103$ & $\$ 20,361$ & $9.5 \%$ & $2.4 \%$ & $100.0 \%$ & $31.1 \%$ & - & $19.7 \%$ \\
\hline Liver & $\$ 78,706$ & $\$ 41,852$ & $0.9 \%$ & $1.2 \%$ & $100.0 \%$ & $58.6 \%$ & - & $0.0 \%$ \\
\hline ESRD+ & $\$ 79,724$ & $\$ 84,996$ & $4.8 \%$ & $1.6 \%$ & $100.0 \%$ & $100.0 \%$ & - & $25.8 \%$ \\
\hline Oncology & $\$ 46,240$ & $\$ 17,052$ & $0.8 \%$ & $1.7 \%$ & $100.0 \%$ & $20.0 \%$ & - & $5.5 \%$ \\
\hline Neurologic & $\$ 66,417$ & $\$ 67,295$ & $3.8 \%$ & $1.9 \%$ & $100.0 \%$ & $75.6 \%$ & - & $9.8 \%$ \\
\hline
\end{tabular}

*Mortality not reported for 2014 since patients who died in 2014 were excluded from the analysis (see the "Methods" section). Patients that died in 2015 were included in calculations for other values reported in this table

disorders (neurologic). We found that these subgroups, while identified using only clinical and demographic data, had markedly different patterns of utilization, spending, and mortality.

Taken together, our findings hold important implications for the design and implementation of care management programs. First, these results add to a growing awareness of the heterogeneity of high-cost populations. ${ }^{6,7,11,22,30,31}$ Traditional narratives describe high-cost patient populations as being comprised of individuals with multiple, poorly controlled chronic conditions, often with coincident frailty and behavioral health disorders. ${ }^{4}$ Though we found this description to be true in aggregate (i.e., higher-than-average average rates of comorbidity among high-cost patients), it obscures substantial heterogeneity within the high-cost population. The mixed subgroup (roughly $60 \%$ of patients) more closely resembled the aforementioned narrative, but the remaining $40 \%$ of patients had disparate diagnoses and clinical compositions, often dominated by either a single condition or a single acute event.

The subgroups we identified share some similarities with high-cost patient subgroups identified in other populations. For example, research in FFS Medicare and single-health system populations have described subgroups of high-cost patients similar to the acute/mixed, ${ }^{6,7,30-32}$ ESRD/ESRD+ ${ }^{6,7}$ vascular, ${ }^{6} \mathrm{GIB},{ }^{32}$ complications, ${ }^{33}$ trauma,${ }^{30}$ and neurologic ${ }^{30}$ subgroups in this study. Incomplete overlap suggests that certain high-cost patient subgroups can be generalized across populations, but also that there is significant variability among different populations.

Second, we found disparate patterns of utilization, spending, and mortality across subgroups, suggesting that uniform care management strategies and interventions are likely to be insufficient. Traditional care management approaches - in which nurse care managers or other allied health professionals assist patients with disease management and medication adherence to reduce the risk of destabilization and inpatient utilization ${ }^{4,30}$ hold promise for subgroups with multi-morbidity and persistently high spending (e.g., ESRD and ESRD+). Interestingly, patients in the acute/mixed subgroup were relatively unlikely to remain high-cost $(35.8 \%)$, casting doubt on the efficacy of traditional care management programs in reducing spending in this subgroup, and pointing to the importance of better identifying patients at risk of being persistently high-cost.

Among the other subgroups identified, traditional care management approaches are unlikely to be effective. For example, the neurologic and liver subgroups had among the highest rates of spending and persistently high spending. Within these subgroups, spending was driven predominately by prescription drug costs, indicating that the rational use and pricing of specialty pharmaceuticals may be the most effective strategies for reducing spending. For subgroups defined by acute events (trauma, GIB, complications), there may be limited opportunities to improve care and reduce spending. High rates of mortality among the ESRD, ESRD+, and GIB subgroups should prompt a focus on recognizing, and addressing, life-limiting illness with palliative care and other interventions.

This study has several limitations. First, our study population consisted of patients enrolled in MA plans offered by a single health insurer. As such, the subgroups we identified may not be generalizable to other populations, including other MA or FFS Medicare populations. However, less is known about MA populations, so our analysis begins to fill an important gap. Second, patterns of spending, utilization, and mortality among the clinical phenotypes we identified could be impacted by existing care management programs at CareMore ${ }^{34}$ and, therefore, may not be generalizable to other populations. Third, we did not have access to patient-level data on important social determinants of health (e.g., income, education, social isolation), despite a growing appreciation for the impact of these factors on spending and outcomes, especially among high-cost patients. Finally, we used the OPTICS algorithm for cluster analysis. Different clustering algorithms are likely to produce different results. However, as discussed in an accompanying article, ${ }^{23}$ we believe OPTICS is the optimal algorithm for clustering high-cost patient populations. 
Prior Presentation(s): This study was presented, in part, at AcademyHealth; June 25, 2018; Seattle, WA.

Corresponding Author: Amol S. Navathe, MD, PhD; Department of Medical Ethics and Health Policy University of Pennsylvania Perelman School of Medicine, Philadelphia, PA, USA (e-mail: amol@wharton.upenn.edu).

Funders This study is supported by a grant from the Anthem Public Policy Institute and, in part, under a grant with the Pennsylvania Department of Health. The Department specifically disclaims responsibility for any analyses, interpretations, or conclusions.

\section{Compliance with Ethical Standards:}

This study was approved by the Institutional Review Board of the University of Pennsylvania.

Conflict of Interest: Dr. Navathe reports that he has received grant support from Hawaii Medical Service Association and Oscar Health; personal fees from Navvis and Co., Navigant Inc., Lynx Medical, Indegene Inc., and Sutherland Global Services; personal fees and equity from NavaHealth; serves on the board without compensation for Integrated Services, Inc., speaking fees from the Cleveland Clinic, and honoraria from Elsevier Press. Dr. Linn reports that she has received grant support from Hawaii Medical Service Association. Dr. Jain reports employment by Anthem, Inc.; stock ownership in Anthem, Inc., and honoraria from Elsevier Press. Ms. Kowalski reports employment by Anthem, Inc. and stock ownership in Anthem, Inc. and Amazon. Dr. Powers reports employment by Anthem, Inc. All other authors declare no conflicts of interest.

Publisher's Note Springer Nature remains neutral with regard to jurisdictional claims in published maps and institutional affiliations.

\section{REFERENCES}

1. National Institute of Health Care Management. The Concentration of U.S. Health Care Spending. 2017; https://www.nihcm.org/topics/cost-quality/concentration-of-us-health-care-spending. Accessed October 19 2018.

2. Riley GF. Long-term trends in the concentration of Medicare spending. Health Aff (Millwood). 2007;26(3):808-816.

3. National Academy of Medicine. Effective care for high-need patients. Washington, DC: National Academy of Medicine; 2017.

4. Hong CS, Abrams MK, Ferris TG. Toward increased adoption of complex care management. N Engl J Med. 2014;371(6):491-493.

5. McWilliams JM. Cost Containment and the Tale of Care Coordination. N Engl J Med. 2016;375(23):2218-2220.

6. Clough JD, Riley GF, Cohen M, et al. Patterns of care for clinically distinct segments of high cost Medicare beneficiaries. Healthc (Amst). 2016;4(3):160-165.

7. Joynt KE, Figueroa JF, Beaulieu N, Wild RC, Orav EJ, Jha AK Segmenting high-cost Medicare patients into potentially actionable cohorts. Healthe (Amst). 2017;5(1-2):62-67.

8. Jacobson G, Damico A, Neuman T, Gold M. Medicare Advantage 2017 Spotlight: Enrollment Market Update. 2017; http://files.kff.org/attachment/Issue-Brief-Medicare-Advantage-2017-Spotlight-Enrollment-Market-Update. Accessed October 19, 2018.

9. Hong CS, Siegel AL, Ferris TG. Caring for High-Need, High-Cost Patients: What Makes for a Successful Care Management Program? 2014; https://www.commonwealthfund.org/sites/default/files/documents/_media_files_publications_issue_brief_2014_aug_1764_hong caring_for_high_need_high_cost_patients_ccm_ib.pdf. Accessed October 19, 2018.

10. Blumenthal D, Abrams MK. Tailoring complex care management for high-need, high-cost patients. JAMA. 2016;316(16):1657-1658.

11. Hayes SL, Salzberg CA, McCarthy D, et al. High-Need, High-Cost Patients: Who Are They and How Do They Use Health Care-A
Population-Based Comparison of Demographics, Health Care Use, and Expenditures. 2016; https://www.commonwealthfund.org/sites/default/files/documents/__media_files_publications_issue_brief_2016 aug_1897_hayes_who_are_high_need_high_cost_patients_v2.pdf. Accessed October 19, 2018.

12. Cohen S, Uberoi N. Differentials in the concentration in the level of health expenditures across population subgroups in the U.S., 2010. Statistical Brief \#421 2013; https://meps.ahrq.gov/data_files/publications/st421/stat421.shtml. Accessed October 19, 2018.

13. Zodet M. Characteristics of Persons with High Health Care Expenditures in the U.S. Civilian Noninstitutionalized Population, 2014. Statistical Brief \#496 2016; https://meps.ahrq.gov/data_files/publications/st496/ stat496.pdf. Accessed October 19, 2018.

14. Lynn J, Straube BM, Bell KM, Jencks SF, Kambic RT. Using population segmentation to provide better health care for all: the "Bridges to Health" model. Milbank Q. 2007;85(2):185-208; discussion 209-112.

15. Quan H, Sundararajan V, Halfon P, et al. Coding algorithms for defining comorbidities in ICD-9-CM and ICD-10 administrative data. Med Care. 2005;43(11):1130-1139.

16. Agency for Healthcare Research and Quality. Clinical Classifications Software (CCS) for ICD-9-CM. 2017; https://www.hcup-us.ahrq.gov/ toolssoftware/ccs/ccs.jsp. Accessed October 19, 2018.

17. Agency for Healthcare Research and Quality. Clinical Classifications Software for Services and Procedures. 2017; https://www.hcup-us.ahrq. gov/toolssoftware/ccs_svcsproc/ccssvcproc.jsp. Accessed October 19, 2018.

18. Choudhry NK, Shrank WH, Levin RL, et al. Measuring concurrent adherence to multiple related medications. Am J Manag Care. 2009;15(7):457-464.

19. Agency for Healthcare Research and Quality. Prevention Quality Indicators Overview. 2017; http://www.qualityindicators.ahrq.gov/modules/ pqi_resources.aspx. Accessed October 19, 2018.

20. New York University. Faculty \& Research. 2017; https://wagner.nyu. edu/faculty/billings/nyued-background. Accessed October 19, 2018.

21. Joynt KE, Gawande AA, Orav EJ, Jha AK. Contribution of preventable acute care spending to total spending for high-cost Medicare patients. JAMA. 2013;309(24):2572-2578.

22. Figueroa JF, Joynt Maddox KE, Beaulieu N, Wild RC, Jha AK. Concentration of Potentially Preventable Spending Among High-Cost Medicare Subpopulations: An Observational Study. Ann Intern Med. 2017;167(10):706-713.

23. Yan J, Linn KA, Powers BW, et al. Applying Machine Learning Algorithms to Segment High-Cost Patient Populations. J Gen Intern Med. 2018.

24. Van Der Maaten L. Accelerating t-SNE using tree-based algorithms. J Mach Learn Res. 2014;15(1):3221-3245.

25. Maaten Lvd, Hinton G. Visualizing data using t-SNE. J Mach Learn Res. 2008;9(Nov):2579-2605.

26. Ester M, Kriegel H-P, Sander J, Xu X. A density-based algorithm for discovering clusters a density-based algorithm for discovering clusters in large spatial databases with noise. Proceedings of the Second International Conference on Knowledge Discovery and Data Mining; 1996; Portland, Oregon.

27. Ankerst M, Breunig MM, Kriegel H-P, \#246, Sander r. OPTICS: ordering points to identify the clustering structure. SIGMOD Rec. 1999;28(2):4960.

28. Congressional Budget Office. High-Cost Medicare Beneficiaries. Washington, DC: Congressional Budget Office;2005.

29. The Lewin Group. Individuals Living in the Community with Chronic Conditions and Functional Limitations: A Closer Look. 2010; https:// aspe.hhs.gov/system/files/pdf/75961/closerlook.pdf. Accessed August 21, 2018.

30. Powers BW, Chaguturu SK, Ferris TG. Optimizing high-risk care management. JAMA. 2015;313(8):795-796.

31. Figueroa JF, Jha AK. Approach for achieving effective care for high-need patients. JAMA Intern Med. 2018.

32. Newcomer SR, Steiner JF, Bayliss EA. Identifying subgroups of complex patients with cluster analysis. Am J Manag Care. 2011;17(8):e324-332.

33. Lee NS, Whitman N, Vakharia N, Ph DG, Rothberg MB. High-cost patients: hot-spotters don't explain the half of it. J Gen Intern Med. 2017;32(1):28-34.

34. Hostetter M, Klein S, McCarthy D. CareMore: Improving Outcomes and Controlling Health Care Spending for High-Needs Patients. 2017; https: / / www.commonwealthfund.org/sites/default/files/documents/_media_files_publications_case_study_2017_mar_1937_hostetter_caremore_case_study_v2.pdf. Accessed October 19, 2018. 